\title{
11 What can UNIVAR do?
}

UNIVAR calculates descriptive statistics for univariate data and graphically displays the data as a histogram. Data may be entered at the keyboard or retrieved from a data file created by FILER. It is possible to select a single variable from a bivariate or multivariate file.

UNIVAR can be used to:

(1) List the data on the screen.

(2) Alter individual items (values).

(3) Transform all the items (values). See section 16.

(4) Sort the values in descending order.

(5) Calculate the mean, median, variance, standard deviation, skewness, kurtosis etc. of the data.

(6) Group the data to form a frequency distribution. You can choose the class limits or let the computer make the selection.

(7) Draw a histogram of the data. You can choose the class limits using (6) or let the computer make the selection.

(8) Draw a histogram together with a normal curve.

(9) Calculate the heights (ordinates) of the normal curve.

(10) Calculate the expected frequencies given by the curve. The class limits can be set using (6). Alternatively, the computer can select the class limits.

Data and results can be printed on a printer. The histograms from (7) and (8) can be sent to a printer if you have a suitable graphics dump. See section 19.

Like FILER, UNIVAR loses your original values if you alter your data by editing individual values or applying a transformation. However, unlike FILER, you will not lose your values if you sort them. 